\title{
Arthroscopic Reduction with Endobutton Fixation for Glenoid Fracture
}

\author{
Ettore Taverna ${ }^{1}$ Vincenzo Guarrella ${ }^{1} \quad$ Michael Thomas Freehill ${ }^{2}$ Guido Garavaglia ${ }^{3}$ \\ ${ }^{1}$ Istituto Ortopedico Galeazzi, Milano, Italy \\ ${ }^{2}$ Wake Forest University Baptist Health, Winston-Salem, North \\ Carolina, United States

\begin{abstract}
Address for correspondence Vincenzo Guarrella, MD, IRCCS Istituto Ortopedico Galeazzi, via Riccardo Galeazzi 4, 20161, Milano, Italy (e-mail: vguarrella@hotmail.com).
\end{abstract}

${ }^{3}$ Servizio di Chirurgia dell'Arto Superiore, OBV, Mendrisio, Switzerland

Joints 2017;5:127-130.

\begin{abstract}
Keywords

- glenoid

- fracture

- fixation

- arthroscopy

- Endobutton

- shoulder dislocation

Glenoid rim fractures, accompanied by acute glenohumeral dislocation or subluxation usually results in persistent instability. Traditionally open reduction and internal fixation has been recommended in displaced intra-articular glenoid fractures. However, open reduction is difficult, and it may not be possible to address the associated intraarticular soft-tissue injuries. A few reports of arthroscopic-assisted fixation of these fractures have been recently published. The most anatomic method for addressing an acute glenoid rim lesion is a reduction (either open or arthroscopic) and internal fixation. We are reporting a case of arthroscopic reduction and fixation of a glenoid fracture utilizing Endobuttons with clinical and radiological results at 18 months follow-up.
\end{abstract}

\section{Introduction}

Fractures of the glenoid cavity are intra-articular and can result in considerable morbidity due to chronic instability of the shoulder, degenerative joint disease, or both. ${ }^{1}$ Direct high-energy impact trauma is the most common mechanism of fractures of the scapula and glenoid and therefore results in a high prevalence of associated osseous and soft-tissue injuries.

Glenoid rim fractures, accompanied by acute glenohumeral dislocation or subluxation usually results in persistent instability. ${ }^{1}$ It is widely recognized such glenoid fractures with a large fragment and/or displacement of more than $10 \mathrm{~mm}$, and associated instability, should be treated surgically. ${ }^{1,2}$

Numerous procedures have been described to address the anterior glenoid bone loss. ${ }^{1}$ The most anatomic method for addressing an acute glenoid rim lesion is reduction (either open or arthroscopic) and internal fixation. Utilizing arthroscopy to identify, reduce, and guide fixation of the fragment can reduce the morbidity of an open anterior surgical approach and potential complications from a subscapularis tenotomy.
Cameron $^{3}$ first reported successful arthroscopic reduction and fixation of an anterior glenoid fracture using a cancellous screw inserted through the subscapularis tendon. More recently, Porcellini et $\mathrm{al}^{4}$ and Sugaya et $\mathrm{al}^{2}$ have described a technique utilizing suture anchors for a reduction in an all-arthroscopic fashion.

The purpose of the present study was to describe cases of arthroscopic reduction and fixation of glenoid fracture utilizing Endobuttons.

\section{Case Description}

A 38-year-old, right hand dominant healthy male sustained a fall while skiing, landing on his right upper extremity in an abducted and elevated position. He subjectively reported a glenohumeral dislocation which had spontaneously reduced. He was subsequently immobilized and transferred to the emergency department for evaluation. Radiographs established the presence of a displaced fracture of the anterior glenoid rim. The patient was discharged with temporary immobilization in a sling. A computed tomography (CT) scan performed 7 days after the injury revealed a
DOI https://doi.org/ $10.1055 / \mathrm{s}-0037-1603675$. ISSN 2282-4324.
Copyright @ 2017 Georg Thieme Verlag KG Stuttgart · New York
License terms

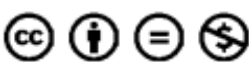




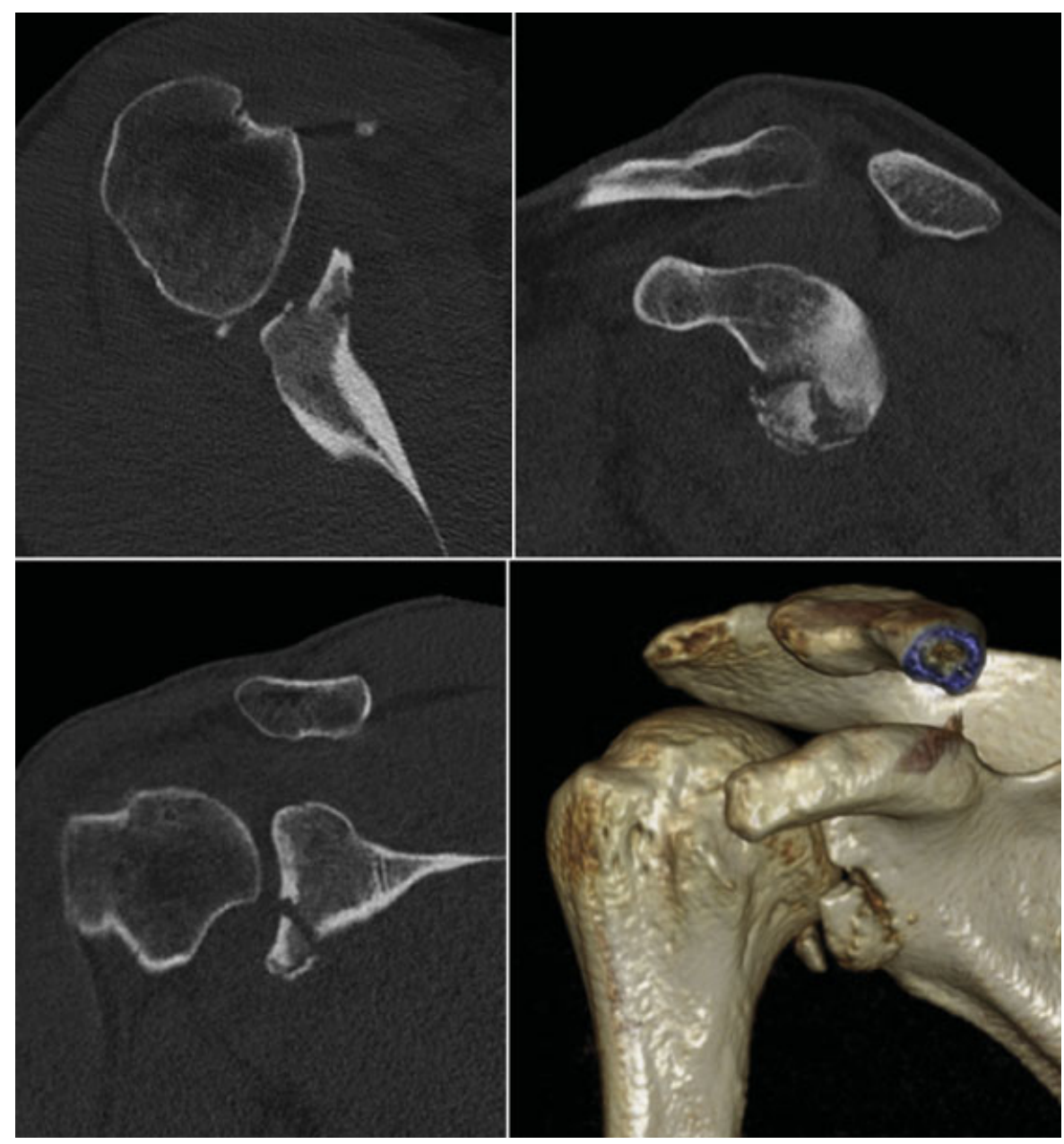

Fig. 1 Preoperative CT scan and 3D reconstruction. 3D, three-dimensional; CT, computed tomography.

displaced glenoid fracture corresponding to roughly $30 \%$ of the glenoid surface (- Fig. 1).

According to the patient's age, the articular incongruity and functional expectations, arthroscopic reduction and internal fixation of the fragment were discussed as a treatment option versus the more invasive open reduction or nonoperative management. The patient accepted the arthroscopic approach and signed a purposed consent with regard to potential risks and complications.

The surgical technique and instrumentation used were similar to those described in an already published article on the arthroscopic bone block procedure. ${ }^{5}$

The arthroscope was introduced into the glenohumeral joint through a standard posterior portal. Diagnostic arthroscopy revealed a large anterior bony fragment with an intact labrum attached. An associated Hill-Sachs lesion was not identified.

Debridement and reduction were performed with the arthroscope positioned through the anterosuperior portal. The anatomical reduction was achieved with the hook of the glenoid guide inserted through the posterior portal and a grasper introduced through an additional low anterior portal.

The glenoid guide allows placements of two parallel drill sleeves at a distance of $10 \mathrm{~mm}$ from each other and $5 \mathrm{~mm}$ off the joint surface. Depending on the size of the bony fragment, a choice is made whether to place one or two couples of round Endobuttons (Double Round Endobutton; Smith \& Nephew, Andover, Massachusetts, United States). In this case, we elected to place a single couple of round Endobuttons in the middle of the fragment and to achieve this, the hook of the guide was placed slightly inferiorly, thus permitting the superior drill to be in the middle of the fracture fragment.

The Double Round Endobutton system was positioned with the standard technique, ${ }^{5}$ and a suture tensioner device was used to secure the implant and provide strong compression at the fracture site.

The rest of the anterior labrum, capsule, and ligaments were reinserted on the glenoid rim using a standard arthroscopic soft-tissue repair technique with suture anchors. We placed drill holes for suture anchors with the cannulated sleeve still in place, because drilling at a later stage when sleeves have been removed would endanger the sutures of the Endobuttons.

The fracture reduction and stability were assessed moving the shoulder in all planes, and the fracture showed an excellent fixation. Probing of the fragment revealed no motion.

Postoperatively, the shoulder was immobilized in an abduction pillow brace of 10 degrees for 3 weeks. At 4 weeks, 


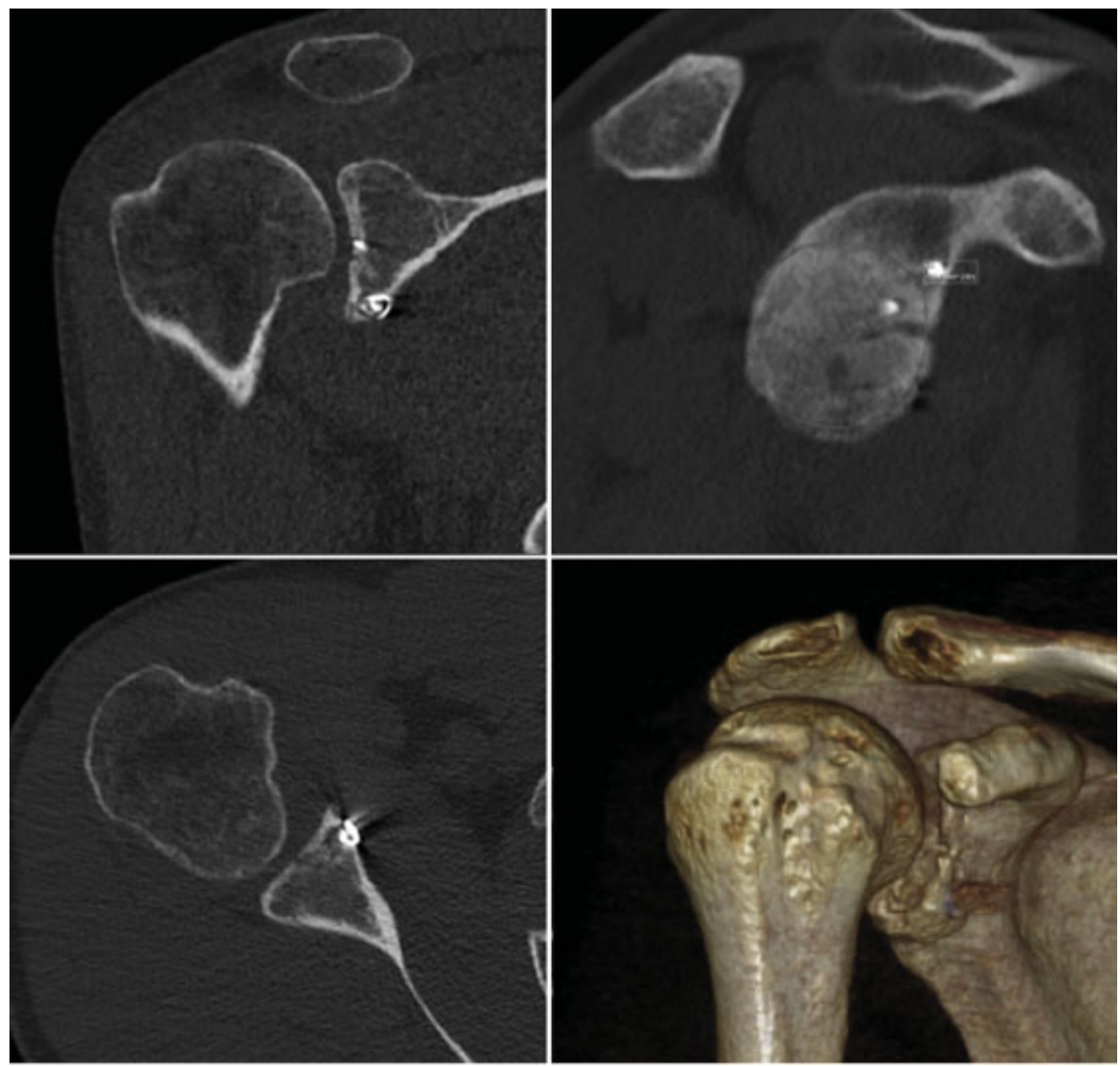

Fig. 2 Postoperative CT scan and 3D reconstruction at 6 months. 3D, three-dimensional; CT, computed tomography.

radiographs showed no displacement of the fracture. No limitations regarding passive movement were prescribed after immobilization, and the patient was subsequently allowed to regain full elevation and external rotation.

After complete healing of the surgical wounds, pool exercises, and return to work activities were authorized. Progressive stretching exercises were started after 6 to 8 weeks. Return to sports and overhead mobility were allowed 5 months after surgery. At 6 months after surgery, the patient reported no pain and was satisfied with his return to preinjury activities. Physical examination revealed full active forward elevation, 60 degrees of external rotation and internal rotation up the posterior thorax to the T10 level. No weakness to resisted external rotation or forward elevation was observed when compared with his contralateral arm, and there were no impingement signs.

Postoperative CT scans demonstrated healing of the bone fragment in an appropriate position (-Fig. 2). The patient has since progressed to all of his previous activity levels. At his last follow-up, 18 months after surgery clinical and radiological complete recovery was confirmed.

\section{Discussion}

Appropriate management of bony pathology in the setting of acute anterior glenohumeral instability is critical to preventing recurrent dislocation. ${ }^{1}$ When a glenoid fracture fragment is present, incorporation of the fragment into the repair construct is recommended. Such repaired fractures heal reliably and reduce the risk of recurrent instability. ${ }^{1}$ However, in the presence of a larger bony fragment, this can be a difficult task to accomplish arthroscopically. Techniques for addressing this problem have been previously reported in the literature. Some authors ${ }^{2,4}$ advocate an arthroscopic anchor-based technique in which sutures are wrapped around the bony fragment and labrum.

Also, a variety of suture-bridge techniques has been reported. ${ }^{1}$ These techniques, however, rely on permanent suture passing over the articular surface of the fracture fragment, which may not be ideal when a large portion of the glenoid is involved.

The technique described in this article is an alternative for reduction with stable fixation of large glenoid fractures. The arthroscopic technique allows close inspection and management of concomitant intra-articular pathology, as well as confirmation of anatomic reduction, additionally avoiding the morbidity and complexity of an open deltopectoral incision with subscapularis detachment or splitting. Transosseous sutures provide secure fixation deep to the articular surface, whereas the soft-tissue repair approximates the labrum, restores tension to the anterior capsular ligaments, and provides additional stability to the bony fragment. By the use of a dedicated tensioner, a good fixation is achieved with strong compression across the fracture site enhancing bone healing. 


\section{References}

1 van Oostveen DP, Temmerman OP, Burger BJ, van Noort A, Robinson M. Glenoid fractures: a review of pathology, classification, treatment and results. Acta Orthop Belg 2014;80(01):88-98

2 Sugaya H, Kon Y, Tsuchiya A. Arthroscopic repair of glenoid fractures using suture anchors. Arthroscopy 2005;21(05):635. Doi:10.1016/j.arthro.2005.02.006
3 Cameron SE. Arthroscopic reduction and internal fixation of an anterior glenoid fracture. Arthroscopy 1998;14(07):743-746

4 Porcellini G, Campi F, Paladini P. Arthroscopic approach to acute bony Bankart lesion. Arthroscopy 2002;18(07):764-769

5 Taverna E, D’Ambrosi R, Perfetti C, Garavaglia G. Arthroscopic bone graft procedure for anterior inferior glenohumeral instability. Arthrosc Tech 2014;3(06):e653-e660 